\title{
Dynamic gene expression profiles during postnatal development of porcine subcutaneous adipose
}

Jie Zhang, Jideng Ma, Keren Long, Long Jin, Yihui Liu, Chaowei Zhou, Shilin Tian, Lei Chen, Zonggang Luo, Qianzi Tang, An'an Jiang, Xun Wang, Dawei Wang, Zhi Jiang, Jinyong Wang, Xuewei Li, Mingzhou Li

A better understanding of the control of lipogenesis is of critical importance for both human and animal physiology. This requires a better knowledge of the changes of gene expression during the process of adipose tissue development. Thus, the objective of the current study was to determine the effects of development on subcutaneous adipose tissue gene expression in growing and adult pigs. Here, we present a comprehensive investigation of mRNA transcriptomes in porcine subcutaneous adipose tissue across four developmental stages using digital gene expression profiling. We identified 3,274 differential expressed genes associated with oxidative stress, immune processes, apoptosis, energy metabolism, insulin stimulus, cell cycle, angiogenesis and translation. A set of universally abundant genes (ATP8, COX2, COX3, ND1, ND2, SCD and TUBA1B) was found across all four developmental stages. This set of genes may play important roles in lipogenesis and development. We also identified development-related gene expression patterns that are linked to the different adipose phenotypes. We showed that genes enriched in significantly up-regulated profiles were associated with phosphorylation and angiogenesis. In contrast, genes enriched in significantly down-regulated profiles were related to cell cycle and cytoskeleton organization, suggesting an important role for these biological processes in adipose growth and development. These results provide a resource for studying adipose development and promote the pig as a model organism for researching the development of human obesity, as well as being used in the pig industry. 


\section{Dynamic Gene Expression Profiles during Postnatal}

\section{Development of Porcine Subcutaneous Adipose}

3 Jie Zhang ${ }^{1,2}$, Jideng Ma ${ }^{1 \bullet}$, Keren Long ${ }^{1}$, Long Jin ${ }^{1}$, Yihui Liu ${ }^{1}$, Chaowei Zhou ${ }^{1,3}$, Shilin Tian ${ }^{4}$, Lei

4 Chen $^{5}$, Zonggang Luo ${ }^{2,5}$, Qianzi Tang ${ }^{1}$, An'an Jiang ${ }^{1}$, Xun Wang ${ }^{1}$, Dawei Wang ${ }^{4}$, Zhi Jiang ${ }^{4}$, Jinyong

5 Wang ${ }^{5}$, Xuewei $\mathrm{Li}^{1 *} \&$ Mingzhou $\mathrm{Li}^{1 *}$

61 Institute of Animal Genetics and Breeding, College of Animal Science and Technology, Sichuan Agricultural

7 University, Ya'an, Sichuan, China,

82 Department of Animal Science, Southwest University at Rongchang, Chongqing, China,

$9{ }^{3}$ Department of Aquaculture, Southwest University at Rongchang, Chongqing, China,

$10{ }^{4}$ Novogene Bioinformatics Institute, Beijing, China,

$11{ }^{5}$ Chongqing Academy of Animal Science, Chongqing, China.

12 These authors contributed equally to this work.

13 * Corresponding author: Xuewei Li, xuewei.li@sicau.edu.cn; Mingzhou Li, mingzhou.li@sicau.edu.cn

14

\section{Abstract}

A better understanding of the control of lipogenesis is of critical importance for both human and animal physiology. This requires a better knowledge of the changes of gene expression during the process of adipose tissue development. Thus, the objective of the current study was to determine the effects of development on subcutaneous adipose tissue gene expression in growing and adult pigs. Here, we present a comprehensive investigation of mRNA transcriptomes in porcine subcutaneous adipose tissue across four developmental stages using digital gene expression profiling. We identified 3,274 differential expressed genes associated with oxidative stress, immune processes, apoptosis, energy metabolism, insulin stimulus, cell cycle, angiogenesis and translation. A set of universally abundant genes (ATP8, COX2, COX3, ND1, ND2, SCD and TUBA1B) was found across all four developmental stages. This set of genes may play important roles in lipogenesis and development. We also identified development-related 
gene expression patterns that are linked to the different adipose phenotypes. We showed that genes enriched in significantly up-regulated profiles were associated with phosphorylation and angiogenesis. In contrast, genes enriched in significantly downregulated profiles were related to cell cycle and cytoskeleton organization, suggesting an important role for these biological processes in adipose growth and development. These results provide a resource for studying adipose development and promote the pig as a model organism for researching the development of human obesity, as well as being used in the pig industry.

\section{Introduction}

Adipose is an anatomical term for loose connective tissue composed of adipocytes (Poissonnet et al., 1988). It is recognized as more than an energy reservoir for lipid storage; in fact, it plays important roles in the pathogenesis of obesity and related comorbidities by secreting cytokines that are involved in the regulation of metabolism (MacDougald \& Burant, 2007; Rosen \& Spiegelman, 2006; Mori et al., 2012). Adipose, unlike most other tissues/organs, grows and develops continuously throughout the lifespan via differentiation of pre-adipocytes into adipocytes, as well as controlling the storage and mobilization of lipids (Boucher et al., 2012; Lijnen et al., 2006), and it is involved in extracellular matrix proteolysis, adipogenesis, and angiogenesis (Crandall et al., 1997). The size of the adipose tissue mass is defined by both adipocyte number (i.e. hyperplastic growth) and size (i.e. hypertrophic growth) (Albright \& Stern, 1998).

Domestic pigs (Sus scrofa) are an attractive model for studying energy metabolism and obesity given that they share similar anatomical and physiological characteristics with humans, including the cardiovascular, urinary, integumentary, digestive systems and devoid of brown fat postnatally (Spurlock \& Gabler, 2008; Swindle et al., 2012). Furthermore, both humans and pigs are prone to the development of obesity and related cardiovascular diseases such as hypertension and atherosclerosis (Koopmans \& Schuurman, 2015). Previous research indicated that rodents and humans have existed 
54

mark differences in metabolism and adipose tissue biology (i.e. adipsin, TNFa, resistin), and pig models can fill the gap (Arner, 2005). They also provide abundant tissue for analyses or repetitive sampling. Pigs also provide the advantage of highly homogeneous genetic backgrounds, the ability to impose a homogeneous feeding regime, and a relatively short generation interval (12 months).

Here, we present a comprehensive survey of gene expression changes in the porcine hypodermal layer of backfat (HLB) across four postnatal developmental stages $(0,30$, 180 days and 7 years after birth, hereafter referred to as birth, $30 \mathrm{~d}, 180 \mathrm{~d}$, and $7 \mathrm{y}$, respectively). These four representative time points cover major morphological and physiological changes in pig growth and development, namely the newborn (birth) and post-weaning periods $(30 \mathrm{~d})$, the point at which peak commercial value for pork yield (180 d) is reached in the modern pig industry (Fisher et al., 2013), through to "middleaged" (7 y) (Peters \& Johnson, 1997; Bhathena et al., 1996). Given that the average life span of a domestic pig tends to be 10-15 years (with each year equivalent to $\sim 5$ years in a human), 7 years represents the midpoint of the pig's life span and is therefore the starting point of age-related deterioration of physiological functions and metabolism (Nair, 2005; Pollack et al., 2002).

We identified genes differentially expressed (DE) during the development process and performed functional enrichment analysis for DE genes. We found co-expressed genes that are highly expressed during development, which are therefore potentially marker genes of this process. We also used a short time-series expression miner (STEM) method to identify sets of DE genes that are linked to different adipose phenotypes. We envision that this study will serve as a valuable resource in adipose studies, and will promote the pig as a model organism for research into the development of human obesity, as well as being highly relevant for the pig industry.

\section{Materials and Methods}

\section{Ethics statement}


81

All research involving animals was conducted according to the Regulations for the Administration of Affairs Concerning Experimental Animals (Ministry of Science and Technology, China, revised in June 2004) and approved by the Institutional Animal Care and Use Committee in College of Animal Science and Technology, Sichuan Agricultural University, Sichuan, China under permit No. DKY-B20110801. Animals were fed according to the nutrition standards by National Research Council (NRC, 1998) and the feeding conditions (including temperature, humidity etc.) were strictly controlled. All animals were fasted $24 \mathrm{~h}$ prior to slaughter and were slaughtered simultaneously, with the exception of newborn animals which were not fasted before slaughter.

\section{Animals and tissues collection}

A total of 12 healthy female Jinhua pigs (a fatty Chinese native breed) were used in this study from four postnatal developmental stages: $0 \mathrm{~d}$ (birth), $30 \mathrm{~d}, 180 \mathrm{~d}$, and $7 \mathrm{y}$. Each stage included three individuals, which were regarded as biological replicates. Venous blood $(50 \mathrm{ml})$ was collected in pre-chilled tubes from each pig immediately before sacrifice. After sacrifice, the HLB, abdominal subcutaneous adipose (ASA), retroperitoneal adipose (RAD), mesenteric adipose (MAD), greater omentum (GOM), longissimus dorsi muscle (LDM), psoas major muscle (PMM), cardiac muscle (CM), liver, spleen, lung and brain were rapidly separated from each carcass, immediately frozen in liquid nitrogen, and stored at $-80^{\circ} \mathrm{C}$ until RNA and DNA extraction.

\section{Measurement of adipose-related phenotype}

Measurements of concentrations of 8 serum-circulating indicators of metabolism and adipocyte volume are from our previous report ( $L i$ et al., 2012). Serum concentrations of total cholesterol (TC), triglycerides (TG), high density lipoprotein (HDL), low density lipoprotein (LDL), very-low density lipoprotein (VLDL), lipoprotein a (Lip-a), apolipoprotein A1 (Apo-A1) and apolipoprotein B (Apo-B) were determined by using CL8000 clinical chemical analyzer (Shimadzu) via standard enzymatic procedures. The adipocyte volume were measured using hematoxylin-eosin (H\&E) staining method. The 
108

109

110

111

112

113

114

115

116

117

118

119

120

121

122

123

124

125

mean diameter of an adipocyte was calculated as the geometric average of the maximum and minimum diameter, and 100 cells were measured for each sample in randomly selected fields. The mean adipocyte volume (V) was obtained according to the following formula: $V=\pi / 6 \Sigma f i D i / \Sigma f i$, where $D i$ is the mean diameter; fi denotes number of cells with that mean diameter $D i$.

\section{RNA and DNA extraction}

Total RNA was extracted from frozen tissues using TRIzol (Invitrogen) and further purified using an RNeasy column (Qiagen) according to the manufacturer's protocol. RNA integrity and concentration were analysis with the Bioanalyzer 2100 (Agilent Technologies). DNA was isolated from frozen tissues using the DNeasy Blood \& Tissue Kit (Qiagen) follow the manufacturer's instructions.

\section{Digital gene expression (DGE) library preparation and sequencing}

The DGE libraries preparation for HLB was carried out using Illumina Gene Expression Sample Prep Kit according to the manufacturer's protocol. In brief, mRNA was isolated from $4 \mu \mathrm{g}$ of total RNA by binding the mRNA to a magnetic Oligo $\mathrm{dT}$ beads. Double strand cDNA were synthesized using Oligo dT beads. Subsequently, the cDNA samples were digested using the restriction enzyme Nlalll, which recognizes and cuts the most 3' "CATG". Once digested into fragments, cDNA were ligated to Illumina adapter 1, which contains a recognition site for enzyme Mmel, and the enzyme Mmel was used to create the $17 \mathrm{bp}$ tag. The Illumina adapter 2 was ligated after Mmel digestion and obtains $21 \mathrm{bp}$ tag derived from a single transcript with Illumina adapters attached to both ends. A 15 cycle PCR was performed with two primers (primer GX1 and primer GX2) that anneal to the ends of the adapters to enrich the adapter-ligated cDNA construct. The amplified cDNA construct was purified from a 6\% TBE PAGE gel. During the QC steps, Agilent 2100 Bioanaylzer and ABI StepOnePlus Real-Time PCR System are used in quantification and qualification of the sample library. Finally, massively parallel sequencing by synthesis was performed on the Illumina HiSeq ${ }^{\text {TM }} 2000$. Image 
135

136

137

138

139

140

141

142

143

144

145

146

147

148

149

150

151

152

153

154

155

156

157

158

159

160

161

recognition and base calling were performed using the Illumina Pipeline.

\section{Analysis of DGE libraries}

The raw data were filtered after data processing. The filtering pipeline is, as follows: 1) empty reads removal (reads with only 3' adaptor sequences but no tags, 2) low quality tag removal (tags with unknown sequences, N), 3) removal of tags too long or too short, leaving only those $21 \mathrm{nt}$ in length; 4) removal of tags with a copy number of 1 (probably resulting from a sequence error), and 5) generation of clean tags. The raw datasets have been submitted to NCBI Gene Expression Omnibus database with the accession number GSE46755. All possible CAGT+17 nucleotide tags were created by using sus scrofa UniGene from Ensembl. All clean tags were mapped to the reference sequences (Sscrofa 10.2) and only $1 \mathrm{bp}$ mismatch was allowed. The numbers of mapped clean tags was calculated for each library and were then normalized to transcripts per million tags (TPM). To identify DE genes $(P<0.01)$ for the clustering analysis, we used one-way repeated-measures ANOVA for comparisons. Resulting $P$-values of above tests were corrected with adjusted Bonferroni method (FDR < 0.01, 1,000 permutations).

\section{Measurement of mitochondrion DNA (mtDNA) content}

The relative mtDNA copy number was determined by quantitative PCR (q-PCR) with primers for the mitochondrial ATP synthase 6 (ATP6), cytochrome c oxidase 1 (COX1) and NADH dehydrogenase 1 (ND1) genes. The nuclear encoding gene Glucagon (GCG) was simultaneously used as an endogenous control gene for normalization (see Table $\mathbf{S 1}$ for primer sequences). All reactions were performed in triplicates, and negative controls (without template) were always included. Relative mtDNA copy number per diploid cell $=($ No. of copies of the mtDNA gene $) /($ No. of copies of GCG), data are expressed as mean \pm SD.

\section{Analysis of development-related expression patterns}

Gene expression patterns during the investigated four growth and development stages were identified using STEM software (Ernst et al., 2005; Ernst \& Bar-Joseph, 2006), 
162

163

164

165

166

167

168

169

170

171

172

173

174

175

176

177

178

179

180

181

182

183

184

185

186

187

188

which applied for the clustering, comparing, and visualizing gene expression data from short time series experiments (in general, $\sim 8$ time points or fewer). STEM implements a novel method for clustering short time series expression data that can differentiate between real and random patterns. The expression patterns were judged to be statistically significant between the number of genes expected $\left(n_{(E)}\right)$ and the number of genes assigned $(n)$ and potentially contained genes that were coordinately regulated.

To visualize the correlations between genes within the profiles, we constructed colored heat maps by plotting pair-wise correlation values of expression of all the genes within the profiles was performed with MultiExperiment Viewer (MeV) (Saeed et al., 2003). The average gene expression value of each significant cluster profile was correlated with each of nine obesity phenotypic traits using a non-parametric Spearman rank correlation coefficient with Bonferroni correction.

\section{Gene functional enrichment analysis}

A Gene Ontology (GO) and KEGG pathway enrichment analysis was used DAVID (Database for Annotation, Visualization and Integrated Discovery) web server (http://david.abcc.ncifcrf.gov/) (Huang et al., 2008). The $P$ values (i.e. EASE score), which indicated the significance of the comparison, was calculated by Benjaminicorrected modified Fisher's exact test. Only GO and pathway categories with a $P$ value less than 0.05 were considered as significant and listed.

\section{DE genes in QTLs region}

QTL data were downloaded from the Pig Quantitative Trait Locus database (PigQTLdb: http://www.animalgenome.org/QTLdb/pig.html) website (Hu et al., 2013). PigQTLdb release 23 (Apr 21, 2014) contains 10,497 QTLs from 416 publications representing 647 different pig traits. Here, we defined QTL genes as those that have an overlapping region with QTL regions, and the overlapping region is at least half the length of the gene or the QTL region, whichever is shorter. In this study, $\sim 282.57 \mathrm{Mb}$ QTL regions of the 2,311 genes were used for analysis. These were assembled from 
189

190

191

192

193

194

195

196

197

198

199

200

201

202

203

204

205

206

207

208

209

210

211

212

213

214

215

901 high confidence and narrowed (<2 Mb) QTL affecting fatness and fat composition.

\section{q-PCR validation}

Total RNA were treated with RNase-free DNase I (TaKaRa). cDNA synthesis and qPCR was performed using the SYBR ${ }^{\circledR}$ Prime- Script ${ }^{\circledR}$ RT-PCR Kit (TaKaRa) on a CFX96 Real-Time PCR detection system (Bio-Rad). The PCR conditions were 5 min at $42{ }^{\circ} \mathrm{C}, 10$ $\mathrm{s}$ at $95^{\circ} \mathrm{C}$, and then 40 cycles of $5 \mathrm{~s}$ at $95^{\circ} \mathrm{C}$ and $30 \mathrm{~s}$ at $65^{\circ} \mathrm{C}$. The primers of 12 genes (TUBA1B, SCD, COX1, COX2, COX3, ATP8, CYTB, ND1, ND2, ND3, ND4, and ND5) designed for q-PCR analyses are listed in Table S1. q-PCR for each RNA sample was performed in triplicate. $\beta$ actin (ACTB), TATA box binding protein (TBP) and topoisomerase II $\beta$ (TOP2B) genes were simultaneously used as an internal gene for normalization. The mRNA expression was quantified using the $2^{-\Delta \Delta C t}$ method, data are expressed as mean \pm SD.

\section{Results and Discussion}

\section{Phenotypic measurements}

As shown in Fig. 1, the body weight (Mann-Whitney $U$ test, $P<10^{-6}$ ) and adipocyte volumes (Student's $t$-test, $P<10^{-4}$ ) were significantly different among the four stages. Additionally, measurement of eight representative serum adipose metabolism indicators gave the same ranking (One-way ANOVA, $P<0.05$, Fig. S1). These phenotypic differences at various stages of HLB imply the existence of intrinsic molecular differences.

\section{Analysis of DGE profiling libraries}

To investigate gene expression changes during development, 12 porcine HLB DGE libraries were constructed using Illumina DGE methods. These DGE libraries generated 3.66 to 6.5 million raw tags for each of the 12 libraries. After filtering, the total number of clean tags per library produced ranged from 3.32 to 6.04 million and the number of distinct clean tags ranged from 141,865 to 270,124 (Table S2). To estimate the quality of the DGE data, the saturation and distribution of clean tag expression was analyzed (Figs. 
216

217

218

219

220

221

222

223

224

225

226

227

228

229

230

231

232

233

234

235

236

237

238

239

240

241

242

S2-S4).

For tag mapping, one reference tag database that included 22,293 sequences from Ensembl Sscrofa 10.2 was preprocessed. We obtained 177,693 total reference tag sequences and 164,561 unambiguous tag sequences. Tolerances were set to allow one mismatch in each alignment to take into account polymorphisms across samples. Among the distinct tags, the number that could be mapped to genes ranged from 54,177 to 69,786 (Table S2). A total of 12,618 genes were identified, with at least one tag in all analyzed samples. The level of gene expression was further analyzed by calculating the number of unambiguous tags for each gene and then normalizing this to the number of TPM. The results showed that the majority of genes transcribed were represented by fewer than 10 copies and only a small proportion of genes were highly expressed (Fig. S5). This result suggests that many genes have housekeeping cellular roles (and therefore low expression) and these may be the main regulatory mRNAs in adipogenesis and basal cellular metabolism (Guo \& Liao, 2000).

Specifically, a set of genes (ATP8: ATP synthase protein 8; COX2: Cytochrome c oxidase 2; COX3: Cytochrome c oxidase 3; ND1: NADH dehydrogenase 1; ND2: NADH dehydrogenase 2; SCD: Stearoyl-coenzyme A desaturase; TUBA1B: tubulin $\alpha 1 \mathrm{~b})$ shared by the four libraries showed over 100-fold increased expression compared with the average level (total expression of all genes/gene number) in each library, which suggests essential roles for these genes in the growth and development of adipose tissue. Stearoyl-coenzyme A desaturase, which catalyzes the de novo synthesis of monounsaturated from saturated fatty acids, contributes to adipocyte differentiation by synthesizing monounsaturated fatty acids that are incorporated into the triglycerides of the mature adipocytes (Dobrzyn \& Ntambi, 2005). Expression of the SCD gene increased gradually during porcine adipocyte differentiation, indicating a significant role of SCD in this process (Wang et al., 2004). In addition, increased SCD gene expression is associated with obesity (Jones et al., 1996) and elevated SCD gene activity in adipose 
243 tissue is closely related to insulin resistance (Sjögren et al., 2008). Furthermore, the SCD 244 gene has served as a postnatal marker of adipocyte differentiation in bovine 245 subcutaneous adipose tissue (Martin et al., 1999). The TUBA1B gene has been shown 246 to play an important role in the metabolic actions of insulin, and a mutation in this gene

has been associated with insulin resistance (Kapeller et al., 1995). Cytochrome c oxidase and NADH dehydrogenase showed increased gene expression levels during adipocyte differentiation and intramuscular fat deposition, suggesting that these genes play important roles in fat development (Lee et al., 2012). Deficiencies in COX1 and COX2 gene activity have been correlated with a number of human aging diseases such as Alzheimer's and muscle atrophy (Barrientos et al., 2002). Obese mice also have higher COX activity levels than lean mice (Mercer \& Trayhurn, 1987; Hebert et al., 2003). In addition, the mitochondrial ATP8 mutation induces mitochondrial reactive oxygen species (ROS) generation, secretory dysfunction, and $\beta$-cell mass adaptation in mice and has been associated with type-2 diabetes and obesity (Weiss et al., 2012).

The expression of these genes was validated to ensure the changes were real, and not caused by technical error. q-PCR was applied to investigate the relative expression levels of these genes, and five other genes, in each of the four developmental stages. We correlated the DGE and q-PCR results, which showed that the expression patterns of these genes were consistent with each other (Pearson's $r>0.7, P<0.05$; Fig. S6). The fold-change in expression among the stages was often lower when assessed by q-PCR compared with DGE profiling; however, these differences may derive from the intrinsic differences between these two technology platforms (Linsen et al., 2009).

\section{Analysis of DE genes}

To explore global transcriptional changes, we identified 3,274 DE genes across the four developmental stages. Gene Ontology (GO) and pathway analysis revealed that DE genes were significantly enriched in the oxidative stress, immune process, apoptosis, energy metabolism process, hormone stimulus, cell cycle, angiogenic and translation categories (Fig. S7), such as 'response to oxidative stress' ( $P=1.74 \times 10^{-4}, 40$ genes), 
271 'regulation of immune system process' ( $P=3.19 \times 10^{-4}, 52$ genes), 'leukocyte activation' $272\left(P=4.43 \times 10^{-3}, 48\right.$ genes $)$, 'regulation of apoptosis' $\left(P=1.94 \times 10^{-2}, 128\right.$ genes $)$, 273 'phosphorylation' ( $P=1.65 \times 10^{-2}, 128$ genes), 'response to insulin stimulus' $(P=1.09 \times$ $27410^{-2}, 23$ genes), 'blood vessel development' $\left(P=2.21 \times 10^{-2}, 45\right.$ genes $)$ and 275 'transcription factor binding' ( $P=4.47 \times 10^{-2}, 83$ genes). Oxidative stress accumulating 276 over a lifetime can inflict direct cellular damage and influence various signaling pathways 277 and transcriptional programs regulating key development processes including immunity,

278

279

280

281

282

283

284

285

286

287

288

289

290

291

292

293

294

295

296

297 energy metabolism, and apoptosis (Finkel \& Holbrook, 2000). This suggests that interactions between biological processes affect porcine adipose development and growth. The increased levels of ROS observed in aging adipose tissue are likely a reflection of the modulatory action of age-related levels of hypoxia on adipose function. Hypoxia increases insulin-stimulated glucose uptake and decreases the lipid content of differentiated 3T3-L1 cells (Zhang et al., 2011). In addition, aging-associated oxidative stress reduces the fat mass in mice, while in vitro studies have found that increased oxidative stress through glutathione depletion alters pre-adipocyte differentiation (Zhang et al., 2011). Adipose tissue growth comprises of the enlargement of existing adipocytes and the formation of new cells from committed pre-adipocytes. This is accompanied by the coordinated development of a well-defined vascular system, which ensures that every adipocyte is surrounded by one or more capillaries. Angiogenesis plays an important role in adipose development not only by supplying nutrients and oxygen to nourish adipocytes, but also as a cellular reservoir of adipose precursor and stem cells that control adipose tissue mass and function (Cao, 2013). As part of the immune system, adipose tissue has also been identified to be involved in aging-associated changes in immune and inflammatory responses. Aged visceral fat in mice showed higher levels of the pro-inflammatory cytokines IL-1 $\beta$, IL-6, TNF- $\alpha$ and COX-2, but lower expression of the anti-inflammatory PPAR-y, chemokine and chemokine receptor (Wu et al., 2007; Lumeng et al., 2011). Starr et al. revealed that IL-6 increased in white adipose tissue with 
298

299

300

301

302

303

304

305

306

307

308

309

310

311

312

313

314

315

316

317

318

319

320

321

322

323

324

age (Starr et al., 2009). The particular role of adipose tissue in calorie storage makes adipocytes well suited to the regulation of energy balance. Some adipocyte-secreted proteins, including leptin, adiponectin and resistin, play important roles in regulating whole-body metabolism (Galic et al., 2010; Kershaw \& Flier, 2004). To meet the energy requirements of fat and other tissue development, adipocytes allocate energy by responding to hormones, cytokines, and other factors that are involved in energy metabolism (Frühbeck et al., 2001; Klaus, 2004).

To evaluate the reproducibility of DGE library sequencing, we analyzed the expression variations of the $3,274 \mathrm{DE}$ genes by hierarchical clustering analysis to indicate the reliability of our experimental results as well as operational stability. As shown in Fig. 2A, the three biological replicates were highly correlated with each other (average Pearson's $r=0.972, P<10^{-16}$, Fig. S8) and all individuals could be clearly assigned to a group, suggesting experimental reliability and further highlighting the low variation in HLB mRNA profiles across different individuals. Interesting, the mRNA expression profiles show an obvious development-specific pattern. Two major branches are defined: one representing birth, $30 \mathrm{~d}$ and $180 \mathrm{~d}$, and one representing $7 \mathrm{y}$. This different clustering pattern may correspond to the intrinsically different biochemical and physiological properties of porcine adipose tissue. Principal components analysis clearly recapitulated these findings. Furthermore, within the treatment groups, gene expression varied significantly by development and time point, as depicted by the well-defined clustering of treatment groups (Fig. 2B). Our previous comparative study of methylation and gene expression profiles of $180 \mathrm{~d}$ and $7 \mathrm{y}$ porcine skeletal muscle indicated that in the aged (7 y) pig, symptoms of muscle atrophy have emerged, suggesting that porcine adipose tissue at this point may appear distinct or show specific gene expression patterns compared with earlier stages of growth and development (Jin et al., 2014); this also supports the findings of our current study.

We also examined the chromosomal distributions of the 3,274 DE genes, but did not 
325

326

327

328

329

330

331

332

333

334

335

336

337

338

339

340

341

342

343

344

345

346

347

348

349

350

351

find over- or under-representation of highly DE genes within a particular chromosome, except for the mitochondrion $\left(P<10^{-4}, \chi^{2}\right.$-test, Table 1). Through BLAST analysis of the 3,274 DE gene sets against high confidence and narrowed ( $<2 \mathrm{Mb}$ ) QTLs affecting fatness and fat composition in the PigQTLdb (Hu et al., 2013), 327 (14.62\%) genes were shown to overlap with the defined DE genes (Table 1). This highlights the potential of identifying candidate genes that may be involved in lipogenesis, development and growth. One such gene is the pig complement factor B (CFB) gene, which was localized to chromosome 7 within the interval of QTLs for backfat thickness, backfat weight, fat area and percentage of backfat in the carcass (Bidanel et al., 2001; Malek et al., 2001; Milan et al., 2002; Gilbert et al., 2007). Another gene is activating transcription factor 6 (ATF6), which was located in the QTL region for body weight, backfat thickness, backfat weight, and linoleic acid percentage (Horak et al., 2010; Fontanesi et al., 2012). Meanwhile, obesity is associated with induction of the endoplasmic reticulum stress response signaling and that ATF6 is the important genes/proteins involved in the signaling regulation (Agouni et al., 2011), which supports the notion that pig might be a good model for further adipose studies.

\section{DE genes involved in inflammatory and lipogenesis process}

Numerous evidence indicates that inflammation is a possible underlying basis for the molecular alterations that link aging and age-related pathological processes (Chung et al., 2006). Based on the annotation of the Pathway Central database (SABiosciences, MD, USA), we identified 38 orthologous inflammation-related genes between pig and human, of which only five genes $(5 / 38,13.15 \%)$ were DE across porcine distinct developmental stages, indicating that there was no obvious change in porcine HLB tissue with the aspect of inflammation till middle age (7 y). Nonetheless, the expression level of two inflammatory genes, CRP and IL13RA1, which plays an important role in anti-inflammatory (Marnell et al., 2005; Alfarsi et al., 2014), have been increasing along with development (Fig. S9A), which may serve as early biomarkers of aging-related 
352 inflammation. In addition, the expression of other three inflammatory genes, $C X 3 C R 1$,

353

354

355

356

357

358

359

360

361

362

363

364

365

366

367

368

369

370

371

372

373

374

375

376

377

378

CXCL9 and IL1ORA, showed a steady increase during birth to $180 \mathrm{~d}$ followed the sharp decrease in 7 y (Fig. S9B), which also need to pay attention in further aging related studies.

Furthermore, previous studies in human have identified several key candidate genes involved in lipid metabolism, such as FASN, PPARG, CEBPA, SLC1A4, ADIPOR1 and ADIPOR2 genes (Wang et al., 2004; Kersten et al., 2000; Yamamoto et al., 2010). Surprisingly, these genes in our study were not DE across porcine distinct developmental stages in HLB tissue, however, they also showed a trend consistent of their biological function. Lipogenesis genes were high expression in $30 \mathrm{~d}$ and $180 \mathrm{~d}$ (Fig. S9C), in contrast, lipolysis genes were low expression in $30 \mathrm{~d}$ and $180 \mathrm{~d}$ (Fig. S9D). Of course, we also found DE genes related lipogenesis, such as CEBPG, CEBPZ and PRKAR1A genes that had higher expression level in Birth than other stages (Fig. S9E), and LIPA, HEXB and ACE genes in $180 \mathrm{~d}$ had highest expression level across four stages (Fig. S9F), suggesting that the governance of lipogenesis in distinct developmental stage are different genes.

\section{Analysis of STEM cluster}

To explore temporal gene expression patterns, we used the STEM algorithm to search the most probable set of clusters generating the observed time series. The 3,274 DE genes were clustered into 24 distinct expression patterns, of which 1,097 DE genes were significantly over-enriched in six expression patterns (Fig. 3A), which have significantly more genes assigned under the true ordering of four stages than the average number assigned to the model profile in the permutation runs. To investigate whether the change of significantly clustered genes affected phenotype variation, we averaged the expression of each significant cluster profile and performed association analysis with the phenotypic traits (Fig. 3B). We identified that these significant cluster profiles significantly correlated with the amount of Lip-a (Spearman $r=0.81, P=1.61 \times 10^{-3}$ ), 
379

380

381

382

383

384

385

386

387

VLDL (Spearman $r=0.78, P=2.59 \times 10^{-3}$; Spearman $r=-0.82, P=1.14 \times 10^{-3}$ ), TG (Spearman $r=0.83, P=7.85 \times 10^{-4} ;$ Spearman $r=-0.85, P=5.21 \times 10^{-4}$ ), LDL (Spearman $\left.r=0.79, P=1.9 \times 10^{-3}\right)$ and TC (Spearman $\left.r=-0.79, P=2.33 \times 10^{-3}\right)$ in serum.

As shown in Fig. 3C, we found that profiles 1, 2, 3 and 4 are highly positive correlated to each other, as are profiles 5 and 6 . We therefore combined these genes for further functional enrichment analysis. Significant GO categories of up-regulated genes (profile 5 and 6) correlated with phosphorylation and angiogenesis (Fig. 3D). Various wellknown genes involved in phosphorylation and angiogenesis of adipose were identified. For example, pyruvate dehydrogenase kinase 2 (PDK2) and pyruvate carboxylase (PC) are involved in phosphorylation, which continually increased with adipose growth and development (Fig. S10), and may play a role in diabetes, lipogenesis and insulin secretion (Lee, 2014; Xu et al., 2008). Angiopoietin 2 (ANGPT2) and epidermal growth factor-like domain 7 (EGFL7) are increased with adipose growth and development in general (Fig. S10), and could be regulating vasculogenesis (Wang et al., 2014; Bambino et al., 2014). These results are consistent with these genes and their associated processes having important roles in the process of adipose development. Significant GO categories of down-regulated genes (profiles 1, 2, 3 and 4) correlated with cell cycle and cytoskeleton organization (Fig. 3D), consistent with the knowledge that inhibition of the cell cycle occurs during the later stages of growth and development. Genes involved in cell cycle were identified. For example, minichromosome maintenance complex component 2 and 3 (MCM2 and MCM3), which are involved in the initiation of eukaryotic genome replication (Kang et al., 2014); cyclin D3 (CCND3), which functions as a regulator of CDK kinases, whose activity is required for cell cycle G1/S transition (Li et al., 2013); F-box protein 5 (FBXO5), which is a mitotic regulator that interacts with CDC20 and inhibits the anaphase promoting complex (Reimann et al., 2001); both of those genes are decreased with adipose growth and development (Fig. S10).

\section{DE genes in middle-aged pig}


407

408

409

410

411

412

413

414

415

416

417

418

419

420

421

422

423

424

425

426

427

428

429

430

431

432

433

434

The results of STEM analysis revealed that gene expression patterns are up- or downregulated in general; however, the gene expression pattern was changed between $180 \mathrm{~d}$ and $7 \mathrm{y}$ in profile 3 , suggesting that the growth and development of $7 \mathrm{y}$ porcine adipose tissue represents a distinct change. Previous studies mainly carried out in pigs are in neonatal or before they reach the age of 1 year (Zhang et al., 2013; Zhou et al., 2013). Limited studies have been carried out using young adult pigs (2 years old). Therefore, we used middle-aged pigs ( 7 years old) as representative of slightly older individuals. A comparative study of methylation and gene expression profiles of $180 \mathrm{~d}$ and $7 \mathrm{y}$ porcine skeletal muscles indicated that 7-year-old pigs have emerging symptoms of aging ( $\mathrm{Jin}$ et al., 2014). We therefore investigated whether known age-related genes from the Human Ageing Genomic Resources (HAGR) database (Tacutu et al., 2013) were included in the genes found to be DE ( 7 y vs. birth, 7 y vs. $30 \mathrm{~d}, 7$ y vs. $180 \mathrm{~d}, P<0.01$, Student's $t$-test) in our data set. Within the HAGR, GenAge is a database of 288 genes potentially associated with human age, 61 of which were found to be DE with age in our study $(P=$ 0.017, $\chi^{2}$-test, Table S3). GO analysis revealed that these 61 genes are associated with DNA repair, apoptosis, transcription and immune processes. Various well-known genes were identified, such as insulin-like growth factor 1 (IGF1), and peroxisome proliferator activated receptor a (PPARa), a mechanistic target of rapamycin (MTOR) and uncoupling protein 2 (UCP2).

\section{Highly expressed DE genes were related to mitochondrial energy metabolism}

Surprisingly, five of the highly expressed DE genes were mitochondrial genes. It is well known that energy metabolism is closely related to mitochondrial gene expression (Patti \& Corvera S, 2010). To verify that the mitochondrial genes were not in different stages of DE by chance, we performed a $\chi^{2}$-test and found that out of 13 mitochondrial genes, nine (69.23\% $P<0.05)$ exhibited dynamic expression changes during the postnatal development stages in HLB (Table 1). Moreover, a previous study reported that the mtDNA copy number was positively correlated with energy metabolism (Tang et al., 2000). We therefore used the mtDNA copy number of HLB as a means of partially 
435 reflecting the energy metabolism. The mtDNA copy number of HLB was significantly 436 increased during the process of development (One-way ANOVA, $P=9.24 \times 10^{-4}$ ), which

437

438

439

440

441

442

443

444

445

446

447

448

449

450

451

452

453

454

455

456

457

458

459

460

461

462

was consistent with the expression of mitochondrial genes in our study (Pearson's $r=$ $0.89, P=1.15 \times 10^{-4}$, Fig. 4A).

We also quantified the expression level of six mitochondrial genes in various adipose tissues between $180 \mathrm{~d}$ and $7 \mathrm{y}$. Expression of all six genes was higher in SATs than in VATs $(P<0.05$, Student's $t$-test, Fig. 4B), which was comparable with the relative levels of mtDNA copy number between the two types of adipose tissue $(P<0.01$, Student's $t$ test, Fig. 4C). These results revealed distinct functional properties of SATs and VATs, in which SATs mainly store energy but VATs are involved with inflammatory and immune processes (Ibrahim, 2010). A comprehensive survey of mtDNA copy number across different tissues including brain and muscle was performed. Of all the tissues examined, muscle showed the highest mtDNA copy number (Fig. 4D), which is consistent with the higher energy demand of muscle due to its motor function. The mtDNA copy number of brain was relatively lower than that of muscle, but higher than all the visceral organs and adipose tissues $(P<0.05$, Student's $t$-test, Fig. 4D), suggesting that muscle and brain have the highest energy requirements (Belanger et al., 2011; Magistretti et al., 2013).

\section{Conclusion}

This study reports a comprehensive transcriptome survey of HLB in four developmental stages, based on directly sequenced gene expression. The data set and research here shed new light on gene regulation during adipose tissue development. Most of the genes highly expressed during development were mitochondrial-related genes, suggesting the alteration of oxidative metabolism during development. Pigs are considered a good biomedical model for human developmental studies because they share the same general physiology. Indeed, the candidate age-related genes found in our study included approximately $20 \%$ of the known human age-related genes. Furthermore, of all the candidate genes, some were primarily associated with adiposerelated phenotypes, such as triglyceride and cholesterol metabolism, which suggests 
463 these genes may serve as potential biomedical markers for mammalian adipose tissue 464 development.

\section{Acknowledgments}

466 We thank Yanmei Xie, Hongmei Wang and Lu Bai for help with experiments. We are grateful to 467 Lingjin Xian, Qingzhi Li and Zhiping Mu for assistance with sample collections.

\section{Reference}

Agouni A, Mody N, Owen C, Czopek A, Zimmer D, Bentires-Alj M, Bence KK, Delibegović M. 2011. Liverspecific deletion of protein tyrosine phosphatase (PTP) 1B improves obesity- and pharmacologically induced endoplasmic reticulum stress. The Biochem Journal 438: 369-378.

Albright Al, Stern SJ. 1998. Adipose tissue. Encyclopedia of Sports Medicine and Science. Internet Society for Sport Science. Available at http://sportsci.org (accessed 30 May 2015)

Alfarsi MA, Hamlet SM, Ivanovski S. 2014. Titanium surface hydrophilicity modulates the human macrophage inflammatory cytokine response. Journal of Biomedical Materials Research Part A 102: 6067.

Arner P. 2005. Resistin: yet another adipokine tells us that men are not mice. Diabetologia 48: 2203-2205.

Belanger M, Allaman I, Magistretti PJ. 2011. Brain energy metabolism: focus on astrocyte-neuron metabolic cooperation. Cell Metabolism 14: 724-738.

Boucher J, Mori MA, Lee KY, Smyth G, Liew CW, Macotela Y, Rourk M, Bluher M, Russell SJ, Kahn CR. 2012. Impaired thermogenesis and adipose tissue development in mice with fat-specific disruption of insulin and IGF-1 signalling. Nature Communications 3: 902.

Bhathena S, Berlin E, Johnson W. 1996. The minipig as a model for the study of aging in humans: selective responses of hormones involved in carbohydrate and lipid metabolism in different sexes. Advances in Swine in Biomedical Research. New York: Plenum Press, 571-579. disease. Gene 286: 53-63. 
488 Bidanel JP, Milan D, lannuccelli N, Amigues Y, Boscher MY, Bourgeois F, Caritez JC, Gruand J, Le Roy P, 489 Lagant H, Quintanilla R, Renard C, Gellin J, Ollivier L, Chevalet C. 2001. Detection of quantitative trait $490 \quad$ loci for growth and fatness in pigs. Genetics Selection Evolution 33: 289-309.

491 Bambino K, Lacko LA, Hajjar KA, Stuhlmann H. 2014. Epidermal growth factor-like domain 7 is a marker of 492 the endothelial lineage and active angiogenesis. Genesis 52: 657-670.

Crandall DL, Hausman GJ, Kral JG. 1997. A review of the microcirculation of adipose tissue: anatomic, metabolic, and angiogenic perspectives. Microcirculation 4: 211-232.

Cao Y. 2013. Angiogenesis and Vascular Functions in Modulation of Obesity, Adipose Metabolism, and Insulin Sensitivity. Cell Metabolism 18: 478-489.

Chung HY, Sung B, Jung KJ, Zou Y, Yu BP. 2006. The molecular inflammatory process in aging. Antioxid Redox Signal 8: 572-581.

Dobrzyn A, Ntambi JM. 2005. The role of stearoyl-CoA desaturase in the control of metabolism. Prostaglandins Leukotrienes and Essential Fatty Acids 73: 35-41.

Ernst J, Bar-Joseph Z. 2006. STEM: a tool for the analysis of short time series gene expression data. BMC bioinformatics 7: 191.

Ernst J, Nau GJ, Bar-Joseph Z. 2005. Clustering short time series gene expression data. Bioinformatics 21: i159-i168.

Fisher KD, Scheffler TL, Kasten SC, Reinholt BM, van Eyk GR, Escobar J, Scheffler JM, Gerrard DE. 2013. Energy dense, protein restricted diet increases adiposity and perturbs metabolism in young, genetically lean pigs. PLoS One 8: e72320.

508

Finkel T, Holbrook NJ. 2000. Oxidants, oxidative stress and the biology of ageing. Nature 408: 239-247.

Fontanesi L, Galimberti G, Calò DG, Fronza R, Martelli PL, Scotti E, Colombo M, Schiavo G, Casadio R,

510 Buttazzoni L, Russo V. 2012. Identification and association analysis of several hundred single 511 nucleotide polymorphisms within candidate genes for back fat thickness in Italian Large White pigs 512 using a selective genotyping approach. Journal of Animal Science 90: 2450-2464. 
513 Frühbeck G, Gómez-Ambrosi J, Muruzábal FJ, Burrell MA. 2001. The adipocyte: a model for integration of

514 endocrine and metabolic signaling in energy metabolism regulation. American Journal of Physiology-

$515 \quad$ Endocrinology and Metabolism 280: E827-E847.

516 Guo XM, Liao K. 2000. Analysis of gene expression profile during 3T3-L1 preadipocyte differentiation.

$517 \quad$ Gene 251: 45-53.

518 Galic S, Oakhill JS, Steinberg GR. 2010. Adipose tissue as an endocrine organ. Molecular and Cellular 519 Endocrinology 316: 129-139.

520 Gilbert H, P LER, Milan D, Bidanel JP. 2007. Linked and pleiotropic QTLs influencing carcass composition

521 traits detected on porcine chromosome 7. Genetics Research 89: 65-72.

522 Horák P, Stratil A, Svatonová M, Mastalková L, Patáková J, Van Poucke M, Bartenschlager H, Peelman

523 LJ, Geldermann H. 2010. Polymorphism screening and mapping of nine meat performance-related 524 genes in the pig. Animal Genetics 41: 334-335.

525 Hebert PD, Ratnasingham S, deWaard JR. 2003. Barcoding animal life: cytochrome c oxidase subunit 1 526 divergences among closely related species. Proceeding Biological Sciences 270 Suppl 1: S96-99.

527 Huang DW, Sherman BT, Lempicki RA. 2008. Systematic and integrative analysis of large gene lists using 528 DAVID bioinformatics resources. Nature protocols 4: 44-57.

529 Hu ZL, Park CA, Wu XL, Reecy JM. 2013. Animal QTLdb: an improved database tool for livestock animal 530 QTL/association data dissemination in the post-genome era. Nucleic Acids Research 41: D871-879.

531 Ibrahim MM. 2010. Subcutaneous and visceral adipose tissue: structural and functional differences. 532 Obesity Reviews 11: 11-18.

533 Jones BH, Maher MA, Banz WJ, Zemel MB, Whelan J, Smith PJ, Moustaïd N. 1996. Adipose tissue 534 stearoyl-CoA desaturase mRNA is increased by obesity and decreased by polyunsaturated fatty acids. 535 American Journal of Physiology-Endocrinology and Metabolism 271: E44-E49.

536 Jin L, Jiang Z, Xia YD, Lou PE, Chen L, Wang H, Bai L, Xie Y, Liu Y, Li W, Zhong B, Shen J, Jiang A, Zhu

537 L, Wang J, Li X, Li M. 2014. Genome-wide DNA methylation changes in skeletal muscle between young 538 and middle-aged pigs. BMC Genomics 15: 653. 
539 Kershaw EE, Flier JS. 2004. Adipose tissue as an endocrine organ. Journal of Clinical Endocrinology $540 \quad$ Metabolism 89: 2548-2556.

541 Kersten S, Desvergne B, Wahli W. 2000. Roles of PPARs in health and disease. Nature 405: 421-424.

542 Klaus S. 2004. Adipose tissue as a regulator of energy balance. Current drug targets 5: 241-250.

543 Kapeller R, Toker A, Cantley LC, Carpenter CL. 1995. Phosphoinositide 3-kinase binds constitutively to $544 \alpha / \beta$-tubulin and binds to $y$-tubulin in response to insulin. Journal of Biological Chemistry 270: 2598554525991.

546 Kang S, Warner MD, Bell SP. 2014. Multiple Functions for Mcm2-7 ATPase Motifs during Replication 547 Initiation. Molecular Cell 55: 655-665.

548 Koopmans SJ, Schuurman T. 2015. Considerations on pig models for appetite, metabolic syndrome and 549 obese type 2 diabetes: From food intake to metabolic disease. European Journal of Pharmacology 759: $550 \quad 231-239$.

551 Lijnen HR, Christiaens V, Scroyen I, Voros G, Tjwa M, Tjwa M, Carmeliet P, Collen D. 2006. Impaired 552 adipose tissue development in mice with inactivation of placental growth factor function. Diabetes 55: $553 \quad 2698-2704$.

554 Li MZ, Wu HL, Luo ZG, Xia YD, Guan JQ, Wang T, Gu Y, Chen L, Zhang K, Ma J, Liu Y, Zhong Z, Nie J, 555 Zhou S, Mu Z, Wang X, Qu J, Jing L, Wang H, Huang S, Yi N, Wang Z, Xi D, Wang J, Yin G, Wang L, Li 556 N, Jiang Z, Lang Q, Xiao H, Jiang A, Zhu L, Jiang Y, Tang G, Mai M, Shuai S, Li N, Li K, Wang J, Zhang 557 X, Li Y, Chen H, Gao X, Plastow GS, Beck S, Yang H, Wang J, Wang J, Li X, Li R. 2012. An atlas of 558 DNA methylomes in porcine adipose and muscle tissues. Nature Communications 3: 850.

559 Lee SH, Kim SC, Choi B-H, Lim D, Kim NK, Lee JH, Kim OH, Lee CS, Kim HC, Yang BS, Hong SK. 2012. $560 m t-C O X 1, m t-N D 1$ and CREBP are indicators of intramuscular fat content in Hanwoo (Korean cattle). $561 \quad$ Livestock Science 146: 160-167.

562 Linsen SE, de Wit E, Janssens G, Heater S, Chapman L, Parkin RK, Fritz B, Wyman SK, de Bruijn E, 563 Voest EE, Kuersten S, Tewari M, Cuppen E. 2009. Limitations and possibilities of small RNA digital 564 gene expression profiling. Nature Methods 6: 474-476. 
565 Lee IK. 2014. The role of pyruvate dehydrogenase kinase in diabetes and obesity. Diabetes Metabolism 566 Journal 38: 181-186.

567 Lee CK, Weindruch R, Prolla TA. 2000. Gene expression profile of the ageing brain in mice. Nature 568 genetics 25: 294-297.

569 Lumeng CN, Liu J, Geletka L, Delaney C, Delproposto J, Desai A, Oatmen K, Martinez-Santibanez G, 570 Julius A, Garg S, Yung RL. 2011. Aging is associated with an increase in T cells and inflammatory 571 macrophages in visceral adipose tissue. Journal of Immunology 187: 6208-6216.

572 Li ZM, Spagnuolo L, Mensah AA, Rinaldi A, Bhagat G, Mensah AA, Rinaldi A, Bhagat G, Zucca E, Doglioni 573 C, Ferreri AJ, Ponzoni M, Bertoni F. 2013. Gains of CCND3 gene in ocular adnexal MALT lymphomas: 574 an integrated analysis. British Journal of Haematology 160: 719-722.

575 MacDougald OA, Burant CF. 2007. The rapidly expanding family of adipokines. Cell Metabolism 6: 159576161.

577 Marnell L, Mold C, Du Clos TW. 2005. C-reactive protein: ligands, receptors and role in inflammation. $578 \quad$ Clinical immunology 117: 104-111.

579 Mori MA, Raghavan P, Thomou T, Boucher J, Robida-Stubbs S, Macotela Y, Russell SJ, Kirkland JL, 580 Blackwell TK, Kahn CR. 2012. Role of microRNA processing in adipose tissue in stress defense and $581 \quad$ longevity. Cell Metabolism 16: 336-347.

582 Martin GS, Lunt DK, Britain KG, Smith SB. 1999. Postnatal development of stearoyl coenzyme A 583 desaturase gene expression and adiposity in bovine subcutaneous adipose tissue. Journal of Animal $584 \quad$ Science $77: 630-636$.

585 Mercer SW, Trayhurn P. 1987. Effect of high fat diets on energy balance and thermogenesis in brown 586 adipose tissue of lean and genetically obese ob/ob mice. Journal of Nutrition 117: 2147.

587 Malek M, Dekkers JC, Lee HK, Baas TJ, Prusa K, Huff-Lonergan E, Rothschild MF. 2001. A molecular 588 genome scan analysis to identify chromosomal regions influencing economic traits in the pig. II. Meat 589 and muscle composition. Mammalian Genome 12: 637-645. 
590 Milan D, Bidanel JP, lannuccelli N, Riquet J, Amigues Y, Gruand J, Le Roy P, Renard C, Chevalet C. 2002.

591 Detection of quantitative trait loci for carcass composition traits in pigs. Genetics Selection Evolution 34:

$592 \quad 705-728$.

593 Magistretti PJ, Allaman I. 2013. Brain energy metabolism. Neuroscience in the 21st Century. New York:

594 Springer, 1591-1620.

595 Nair KS. 2005. Aging muscle. American Journal of Clinical Nutrition 81: 953-963.

596 Poissonnet CM, LaVelle M, Burdi AR. 1988. Growth and development of adipose tissue. Journal of 597 Pediatrics 113: 1-9.

598 Peters RC, Johnson WA. 1997. Aging and miniature swine heart and liver plasma membranes. Advances 599 in Swine in Biomedical Research. New York: Springer, 581-593.

600 Pollack M, Phaneuf S, Dirks A, Leeuwenburgh C. 2002. The role of apoptosis in the normal aging brain, 601 skeletal muscle, and heart. Annals of the New York Academy of Sciences 959: 93-107.

602 Patti ME, Corvera S. 2010. The role of mitochondria in the pathogenesis of type 2 diabetes. Endocrine 603 Reviews 31: 364-395.

604 Reimann JD, Freed E, Hsu JY, Kramer ER, Peters JM, Jackson PK. 2001. Emi1 is a mitotic regulator that 605 interacts with Cdc20 and inhibits the anaphase promoting complex. Cell 105: 645-655.

606 Rosen ED, Spiegelman BM. 2006. Adipocytes as regulators of energy balance and glucose homeostasis. $607 \quad$ Nature 444: 847-853.

608 Spurlock ME, Gabler NK. 2008. The development of porcine models of obesity and the metabolic 609 syndrome. Journal of Nutrition 138: 397-402.

610 Swindle M, Makin A, Herron A, Clubb F, Frazier K. 2012. Swine as models in biomedical research and 611 toxicology testing. Veterinary Pathology 49: 344-356.

612 Saeed Al, Sharov V, White J, Li J, Liang W, Bhagabati N, Braisted J, Klapa M, Currier T, Thiagarajan M, 613 Sturn A, Snuffin M, Rezantsev A, Popov D, Ryltsov A, Kostukovich E, Borisovsky I, Liu Z, Vinsavich A, 614 Trush V, Quackenbush J. 2003. TM4: a free, open-source system for microarray data management and 615 analysis. Biotechniques 34: 374-378. 
616 Sjögren P, Sierra-Johnson J, Gertow K, Rosell M, Vessby B, de Faire U, Hamsten A, Hellenius ML, Fisher

617 RM. 2008. Fatty acid desaturases in human adipose tissue: relationships between gene expression, 618 desaturation indexes and insulin resistance. Diabetologia 51: 328-335.

619 Starr ME, Evers BM, Saito H. 2009. Age-associated increase in cytokine production during systemic 620 inflammation: adipose tissue as a major source of IL-6. Journal of Gerontology Series A-Biological Sciences and Medical Sciences 64: 723-730.

Tacutu R, Craig T, Budovsky A, Wuttke D, Lehmann G, Budovsky A, Wuttke D, Lehmann G, Taranukha D, databases and tools for the biology and genetics of ageing. Nucleic Acids Research 41: D1027-1033.

Tang Y, Schon EA, Wilichowski E, Vazquez-Memije ME, Davidson E, Davidson E, King MP. 2000. Rearrangements of human mitochondrial DNA (mtDNA): new insights into the regulation of mtDNA copy number and gene expression. Molecular Biology of the Cell 11: 1471-1485.

Wang P, Ko Y, Liu B, Peng H, Lee M, Chen C, Li Y, Ding S. 2004. The expression of porcine adiponectin and stearoyl coenzyme A desaturase genes in differentiating adipocytes. Asian-Australasian Journal of Animal Science 17: 588-593.

Wang, Y, Voy BJ, Urs S, Kim S, Soltani-Bejnood M, Quigley N, Joshi, R. 2004. The human fatty acid synthase gene and de novo lipogenesis are coordinately regulated in human adipose tissue. The Journal of Nutrition 134: 1032-1038.

Weiss H, Wester-Rosenloef L, Koch C, Koch F, Baltrusch S, Tiedge M, Ibrahim S. 2012. The mitochondrial Atp8 mutation induces mitochondrial ROS generation, secretory dysfunction, and beta-cell mass adaptation in conplastic B6-mtFVB mice. Endocrinology 153: 4666-4676.

Wu D, Ren Z, Pae M, Guo W, Cui X, Merrill AH, Meydani SN. 2007. Aging up-regulates expression of inflammatory mediators in mouse adipose tissue. Journal of Immunology 179: 4829-4839.

Wang R, Huebert RC, Shah VH. 2014. Sinusoidal Endothelial Cells Coordinate Liver Regeneration and 640 Angiogenesis via Angiopoietin-2: An Ode to Prometheus. Gastroenterology 147: 533-534. proliferation in rat pancreatic beta cells. Diabetologia 51: 2022-2030. 
643 Yamamoto Y, Gesta S, Lee KY, Tran TT, Saadatirad P, Kahn CR. 2010. Adipose depots possess unique 644 developmental gene signatures. Obesity 18: 872-878.

645 Zhang L, Ebenezer PJ, Dasuri K, Fernandez-Kim SO, Francis J, Mariappan N, Gao Z, Ye J, Bruce-Keller 646 AJ, Keller JN. 2011. Aging is associated with hypoxia and oxidative stress in adipose tissue: 647 implications for adipose function. American Journal of Physiology-Endocrinology and Metabolism 301: $648 \quad$ E599-E607.

649 Zhang J, Zhou CW, Ma JD, Chen L, Jiang AA, Zhu L, Shuai S, Wang J, Li M, Li X. 2013. Breed, sex and 650 anatomical location-specific gene expression profiling of the porcine skeletal muscles. BMC Genetics $651 \quad 14: 53$.

652 Zhou CW, Zhang J, Ma JD, Jiang AA, Tang GQ, Mai M, Zhu L, Bai L, Li M, Li X. 2013. Gene expression 653 profiling reveals distinct features of various porcine adipose tissues. Lipids in Health and Disease 12: 65475.

655

656

657

658

659

660

\section{Figures and legends}


662

663

664

665

666

667

668

669

670

A

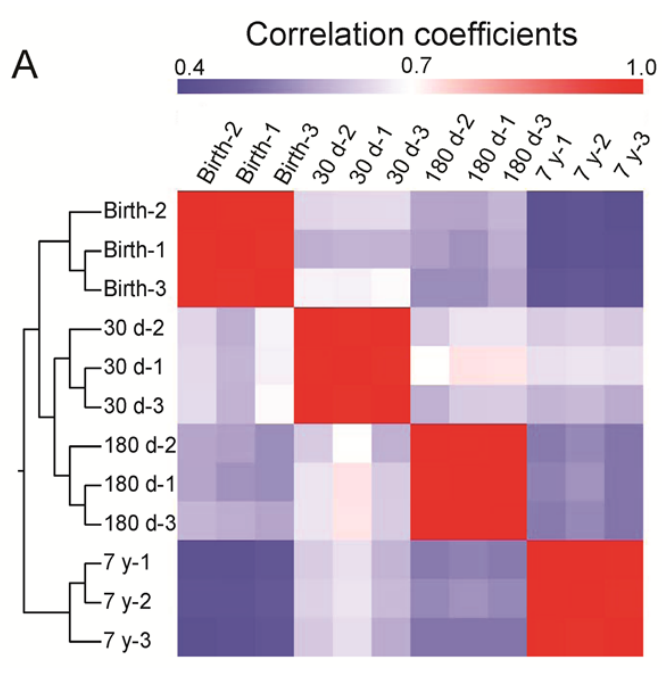

$\mathrm{B}$

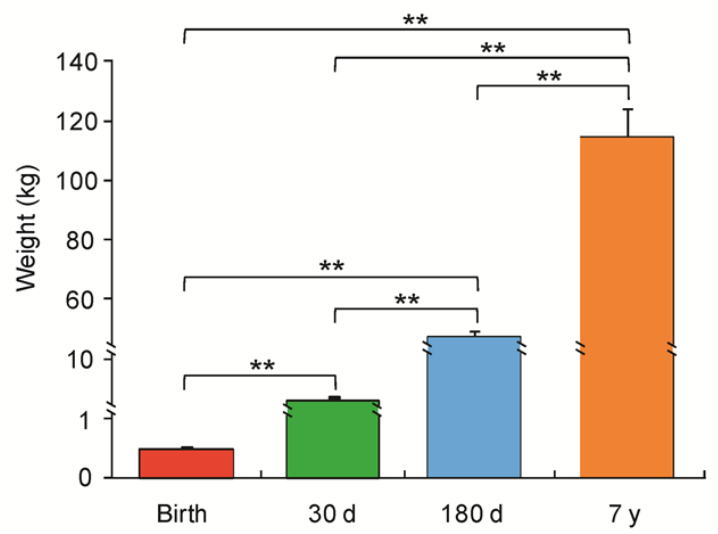

Figure 1. Differences in phenotype. (A) Violin plot of adipocyte volume. Each "violin" with the width depicting a $90^{\circ}$-rotated kernel density trace and its reflection. Vertical black boxes denote the interquartile range (IQR) between the first and third quartiles (25th and 75th percentiles, respectively) and the white point inside denotes the median. Vertical black lines denote the lowest and highest values within 1.5 times IQR from the first and third quartiles, respectively. The statistical significance was calculated by the Mann-Whitney $U$ test $\left({ }^{* *}: P<10^{-6}\right)$. (B) The body weight across four stages. The significance of differences among stages was determined by Student's $t$-test( $\left.{ }^{* *}: P<10^{-4}\right)$.

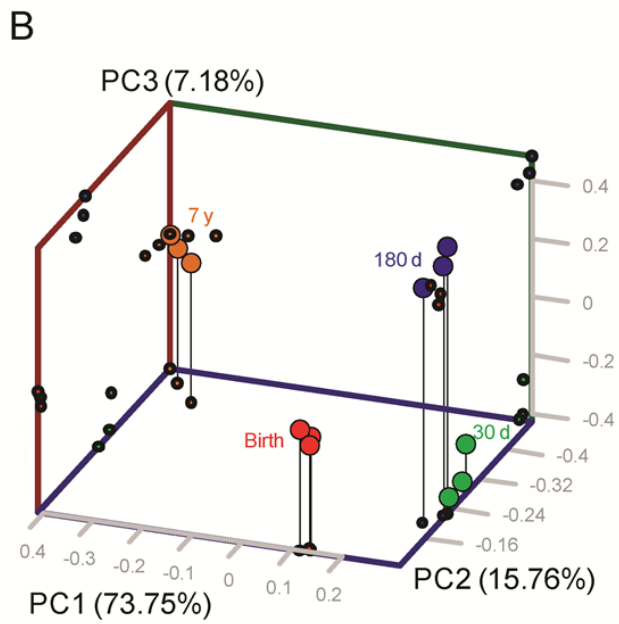

671 Figure 2. Analysis of samples. (A) Heat map matrix of Pearson's correlation among samples. (B)

672 Three-way PCA plot of samples. The fraction of the variance explained is $73.75 \%, 15.76 \%$ and $7.18 \%$ 673 for eigenvector 1,2 and 3. 


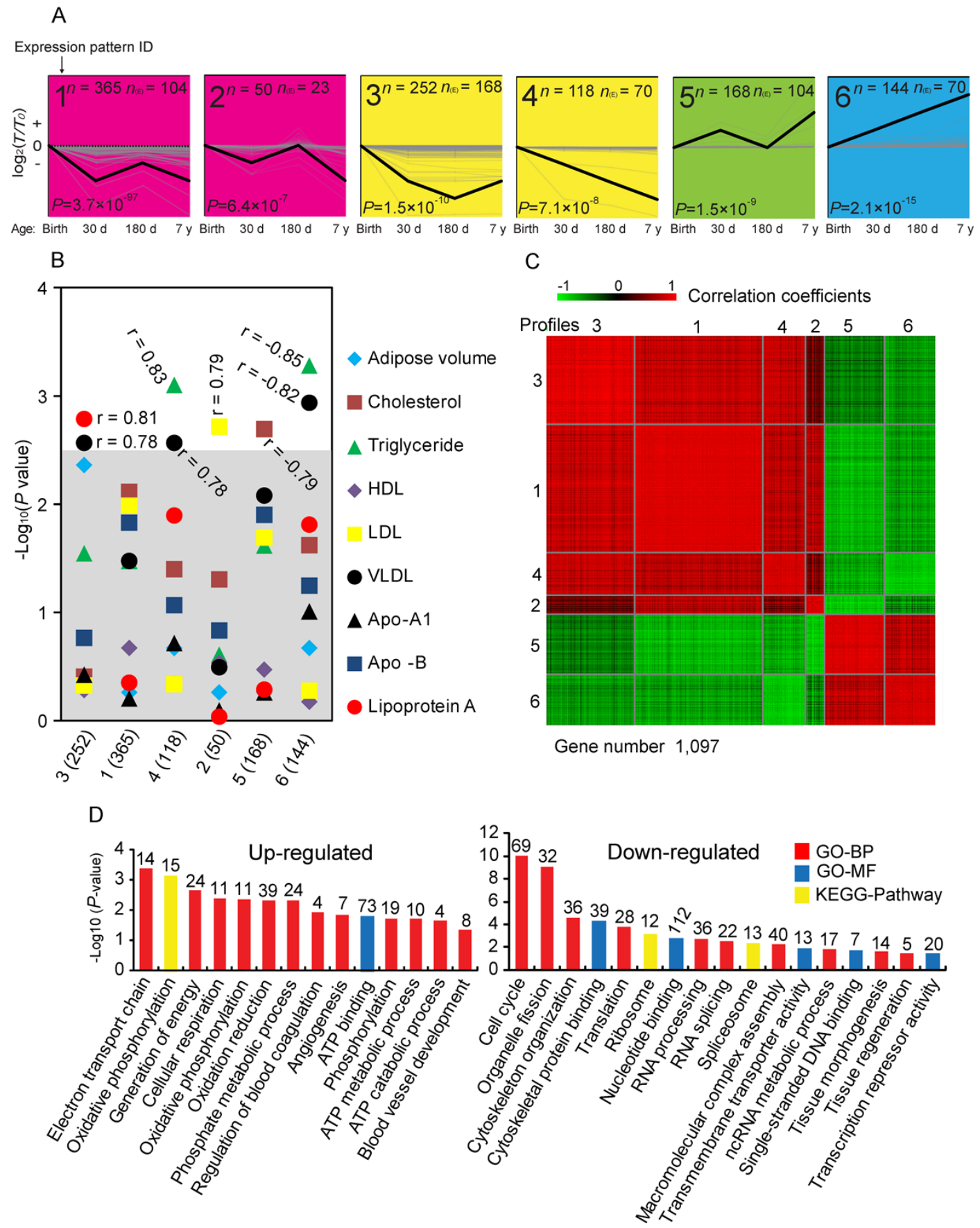

674

675 Figure 3. Analysis of short time-series expression cluster. (A) Six significant cluster profiles that have 676 significantly more genes assigned under the true ordering of time points compared to the average

677 number assigned to the model profile in the permutation runs (Non significant cluster profiles are not 678 shown). The upper left represents the serial number of the cluster, the lower left represents the $P$ 679 value (Fisher's exact test), $n$ represents the number of genes assigned, and $n_{(\mathrm{E})}$ represents the 
680 number of genes expected. Three other expressions were normalized to the highest one in four 681 stages firstly, and then all expressions were $\log _{2}$-transformed. (B) Correlations between significant 682 cluster profiles and phenotypic traits. - Log $P$ values for Spearman correlation coefficients between the 683 values of the profiles and the different phenotypic traits are shown. The gray shadow represents a 684 highly stringent Bonferroni corrected $P$ value of 0.05 . (C) Heat map of significant cluster profiles. 685 Gene pairs strongly positively or negatively correlated are shown in red or green, respectively. (D) 686 Gene Ontology (GO) categories enriched for up- and down-regulated genes involve in significant 687 cluster profiles. The $P$ value, which indicated the significance of the comparison, was calculated by 688 Benjamini-corrected modified Fisher's exact test. BP, biological process; MF, molecular function.

A

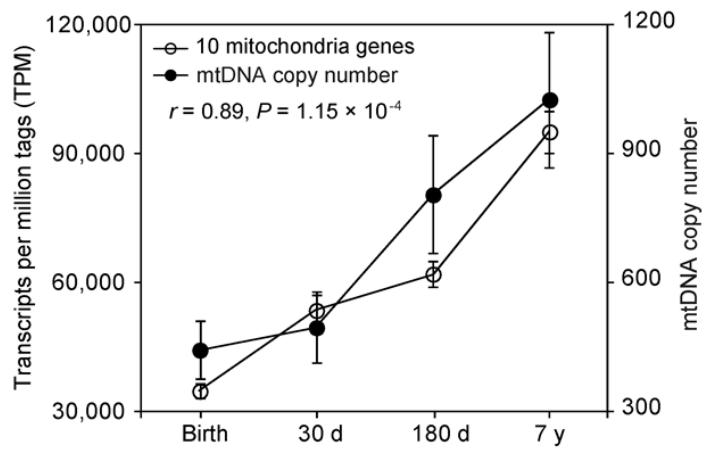

B
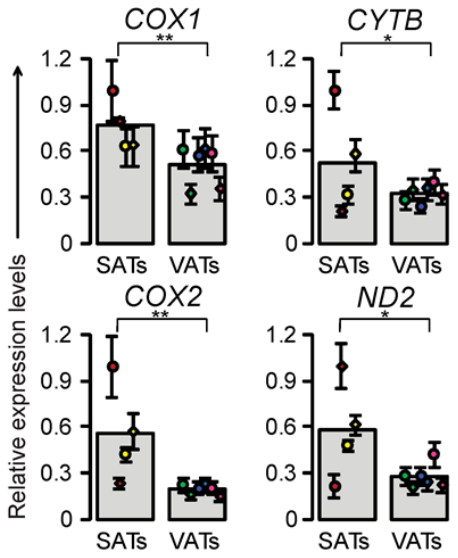

C

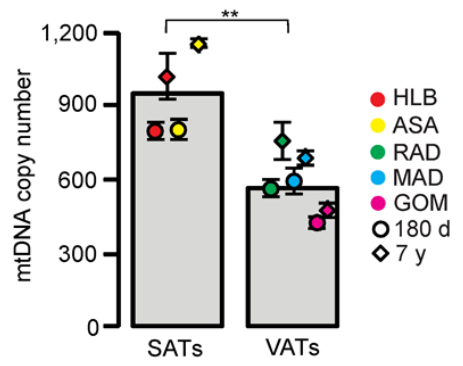

D

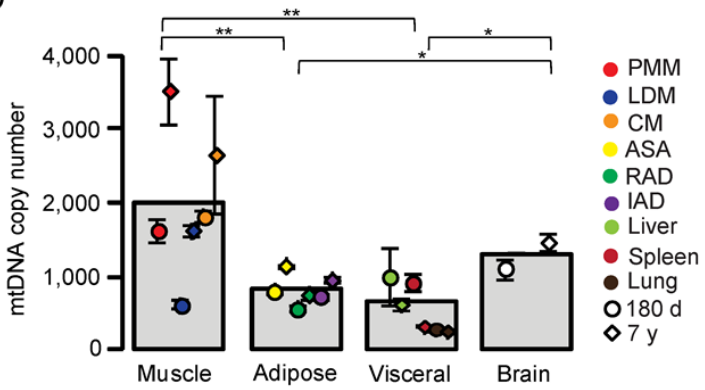

690 Figure 4. Analysis of mitochondrial genes. (A) Correlation of mitochondria genes expression and DNA copy number. Data are means \pm SD. The Pearson correlation coefficient $(r)$ and the corresponding significance value $(P)$ were shown. (B) Comprehensive survey of development stageand tissue- specific expression patterns of mitochondrial genes. SAT: subcutaneous adipose tissue, 
694 VAT: visceral adipose tissue; COX1: cytochrome c oxidase 1, COX2: cytochrome c oxidase 2, CYTB: 695 cytochrome b, ND2: NADH dehydrogenase 2, ND3: NADH dehydrogenase 3 and ND4: NADH 696 dehydrogenase 4. (C) Comprehensive survey of development stage- and tissue- specific 697 mitochondria copy number in various types of adipose tissues. (D) Comprehensive survey of 698 development stage- and tissue- specific mitochondria copy number in various types of tissues. Data 699 are means \pm SD. The significance of differences between samples was determined by Student's $t$ 700 test $\left({ }^{*}: P<0.05 ;{ }^{* *}: P<0.01\right)$.

701

702 Table 1. DE genes distribution in chromosome and QTLs region

\begin{tabular}{ccccc}
\hline $\begin{array}{c}\text { Chromosome/ } \\
\text { Mitochondrion } \\
\text { (Length, Mb) }\end{array}$ & Gene number & $\begin{array}{c}\text { DE gene number } \\
\text { \% of chromosomal } \\
\text { / mitochondrial } \\
\text { background) }\end{array}$ & $\begin{array}{c}\text { DE gene number in } \\
\text { QTLs region }\end{array}$ & $\begin{array}{c}\text { QTLs region } \\
\text { length }(\mathrm{Mb})\end{array}$ \\
\hline $1(315.32)$ & 1,871 & $280(15.97 \%)$ & 28 & 14.98 \\
$2(162.57)$ & 1,935 & $301(15.56 \%)$ & 28 & 9.61 \\
$3(144.79)$ & 1,289 & $192(14.9 \%)$ & 0 & 0 \\
$4(143.47)$ & 1,079 & $178(16 . \%)$ & 55 & 16.02 \\
$5(111.51)$ & 1,035 & $153(14.78 \%)$ & 10 & 3.56 \\
$6(157.77)$ & 1,700 & $282(16.59 \%)$ & 50 & 16.52 \\
$7(134.76)$ & 1,423 & $179(12.58 \%)$ & 38 & 9.47 \\
$8(148.49)$ & 707 & $112(15.84 \%)$ & 15 & 4.52 \\
$9(153.67)$ & 1,224 & $158(12.9 \%)$ & 12 & 4.11 \\
$10(79.10)$ & 426 & $67(0.23 \%)$ & 0 & 0 \\
$11(87.69)$ & 352 & $47(13.35 \%)$ & 3 & 2.01 \\
$12(63.59)$ & 999 & $182(18.22 \%)$ & 11 & 2.82 \\
$13(218.64)$ & 1,298 & $209(16.1 \%)$ & 26 & 11.16 \\
$14(153.85)$ & 1,197 & $207(17.29 \%)$ & 18 & 7.06 \\
$15(157.68)$ & 809 & $138(17.06 \%)$ & 12 & 3.81 \\
$16(86.90)$ & 351 & $53(15.1 \%)$ & 7 & 1.94 \\
$17(69.70)$ & 579 & $99(17.1 \%)$ & 4 & 0.46 \\
$18(61.22)$ & 434 & $69(15.9 \%)$ & 8 & 3.76 \\
$\times(144.29)$ & 733 & $148(20.19 \%)$ & 2 & 0.11 \\
\hline Mitochondrion $(0.0167)$ & 13 & $9 *(69.23 \%)$ & 1 & 1 \\
\hline
\end{tabular}




\section{Supplementary Information}

708 Figure S1. Differences of 8 adipose-related circulating indicators of metabolism in serum. Data normally distributed (Kolmogorov-Smirnov test, $P>0.05$ ). The statistical significance was calculated by one-way repeated-measures ANOVA $(n=3)$. Values are means \pm SD. The Pearson's correlation was calculated between each pair of indicators. The blue and red connecting lines showed positive and negative correlations $\left({ }^{*} P<0.05,{ }^{* *} P<0.01,{ }^{* * *} P<0.001\right)$, respectively. (TIF)

Figure S2. Saturation analysis of DGE libraries. Saturation analysis of the capacity of libraries demonstrated that newly emerging distinct tags were gradually reduced with increase in total sequence tags when the number of sequencing tags was large enough. When the number of sequencing tags reached one million, library capacity approached saturation. (TIF)

Figure S3. Effect of library size on the number of gene identified. The rate of increase of all genes identified and genes identified by unambigous tags declined as the size of the library increased. When the library size reached two million, we could identify $45 \%$ and $35 \%$ of all genes and genes identified by unambigous tags, respectively. At this time, library capacity approached saturation. (TIF) Figure S4. Distribution of total clean tags and distinct clean tags in each library. (A) Distribution of total clean tags. (B) Distribution of distinct clean tags. The distribution of total clean tags and distinct clean tags in each library showed similar patterns; high-expression tags with more than 100 copy numbers comprised $70.1 \%$ of the total clean tags, but their distribution did not even reach $2.96 \%$ of the distinct clean tags, whereas low-expression tags with copy numbers smaller than 5 accounted for more than $70.5 \%$ of the distinct tag distribution. (TIF)

Figure S5. Distribution of gene expression. Gene expression level determined by calculating the number of unambiguous tags and then normalizing to TPM (transcript copies/million tags). (TIF) Figure S6. Validation of gene expression by q-PCR. The data presented in Y-axis indicated the relative mRNA expression of both DGE and q-PCR. Datas are means $\pm \mathrm{SD}$. The Pearson correlation coefficient $(r)$ and the corresponding significance value $(P)$ were shown above the columns. (TIF) 
732 Figure S7. Gene Ontology (GO) categories enriched for DE genes. The EASE score, which indicated 733 the significance of the comparison, was calculated by Benjamini-corrected modified Fisher's exact 734 test. BP, biological process; MF, molecular function. (TIF)

735 Figure S8. Correlation of mRNA expression among three biological replicates within four libraries. A 736 scatter plot and Pearson's correlation revealed a correlation between the $\log _{10}$ of mRNA expression 737 of each biological replicate. (TIF)

738 Figure S9. The expression level of inflammatory and lipogenesis related genes. (TIF)

739 Figure S10. The gene expression level of PDK2, PC, ANGPT2, EGFL7, MCM2, MCM3, CCND3 and 740 FBXO5 genes involved in significant profiles. (TIF)

741 Table S1. Primer sequences used for q-PCR. *: ACTB ( $\beta$ actin), TBP (TATA box binding protein), 742 TOP2B (topoisomerase II $\beta$ ) and GCG (glucagon) are the endogenous control genes. a: Primer 743 sequences used for mRNA q-PCR; b: Primer sequences used for mtDNA copy number q-PCR. (XLS)

744 Table S2. Major characteristics of DGE libraries. Notes: *presents \% of clean tag; **indicates \% of 745 reference genes. (XLS)

746 Table S3. DE genes overlap with known age-related genes. (XLS) 